\title{
Aplicación de materiales orgánicos e inorgánicos en la adsorción de fósforo en un Oxisol
}

\author{
Martha C. D aza-Torres ${ }^{1}$, Javier G. Álvarez-Herrera² \& Jesús H. Camacho-Tamayo³
}

\section{RESU MEN}

Los oxisoles presentan alta fijación de fósforo (P) ocasionada por hidróxidos y óxidos de hierro y aluminio presentes en las arcillas. La aplicación de mezclas de materiales orgánicos e inorgánicos puede ayudar a disminuir dicha fijación. Se utilizaron dos materiales orgánicos (gallinaza y compost) y dos materiales inorgánicos (Cal y Superfosfato triple). Se establecieron un total de trece tratamientos dispuestos en bloques al azar y compuestos por diferentes combinaciones entre fertilizantes minerales y orgánicos. Se determinó la adsorción de P mediante las isotermas de Langmuir, al momento de la siembra y diez semanas después. Todos los tratamientos mostraron que al incrementar la concentración de P, las curvas de adsorción presentaron una tendencia asintótica y se acotaron a un nivel de adsorción máxima, lo que indica la existencia de una quimiadsorción. Al añadir gallinaza en concentraciones menores de $25 \mathrm{mg} \mathrm{L}^{-1}$ de $\mathrm{P}$ se disminuyó la adsorción de $\mathrm{P}$, pero con concentraciones superiores de $75 \mathrm{mg} \mathrm{L}^{-1}$, la retención de $\mathrm{P}$ aumentó. La aplicación combinada de materiales orgánicos y fertilizantes químicos presentó la menor fijación de $\mathrm{P}$, no obstante esta debe hacerse periódicamente, dado que la adsorción de $\mathrm{P}$ volvió a incrementarse, después de 10 semanas.

Palabras-clave: gallinaza, cal, compost, superfosfato triple, quimiadsorción

\section{Application of organic and inorganic materials and phosphorus adsorption in an Oxisol}

\begin{abstract}
The 0 xisols present high fixation of phosphorus (P) caused by oxides and hydroxides of iron and aluminum in the clays. The application of mixtures of organic and inorganic materials can help to decrease this fixation. Two organic materials were used (chicken manure and compost) and two inorganic materials (lime and triple superphosphate). A total of thirteen treatments were established in randomized blocks and composed of different combinations of mineral and organic fertilizers. The adsorption of $\mathrm{P}$ was determined by means of the Langmuir isotherms, at the moment of the crop establishment and ten weeks later. All the treatments showed that when the concentration of $\mathrm{P}$ was increased, the curves of adsorption presented an asymptotic tendency and they were delimited at a level of maximum adsorption, which indicates the existence of chemisorption. When chicken manure was added in concentrations smaller than $25 \mathrm{mg} \mathrm{L}^{-1}$ of $\mathrm{P}$, the adsorption of $\mathrm{P}$ decreased, and when the concentrations were greater than $75 \mathrm{mg} \mathrm{L}^{-1}$ the retention of $\mathrm{P}$ increased. The combined application of organic materials with chemical fertilizers presented the smallest fixation in $\mathrm{P}$, nevertheless it has to be made periodically, because after 10 weeks the $P$ adsorption increased again.
\end{abstract}

Key words: chicken manure, lime, compost, triple superphosphate, chemisorption

\footnotetext{
${ }^{1}$ Escuela de Ingeniería de Recursos N aturales y del Ambiente. Facultad de Ingeniería. Universidad del Valle. Cali. Ciudad Universitaria M eléndez. Ed. 341. Grupo de Investigaciones Agrícolas (GIA). E-mail: conidaza@hotmail.com

2 Facultad de Ciencias Agropecuarias. Universidad Pedagógica y Tecnológica de Colombia. Tunja. Av. Central del Norte. Grupo de Investigaciones Agrícolas (GIA). Email: jgalvarezh@gmail.com

3 Facultad de Ingeniería, Programa de Ingeniería Agrícola, Universidad Nacional de Colombia, Bogotá. Ciudad Universitaria, Ed. 214, Of. 304 . Fone: (+ 57) 13165430. E-mail: jhcamachot@unal.edu.com
} 


\section{INTRODUCCIÓN}

La deficiencia de fósforo $(\mathrm{P})$ en los suelos del trópico y subtrópico seco es un problema de reconocida importancia, cuya magnitud constituye una de las limitantes de mayor trascendencia en la producción de los cultivos. La baja disponibilidad del $\mathrm{P}$ está asociada a bajos contenidos de $\mathrm{P}$ total, alta estabilidad de los compuestos fosfatados del suelo, que implica baja solubilidad del mismo y por tanto una lenta liberación de formas disponibles para la planta y a una baja intensidad de la mineralización de los compuestos orgánicos fosfatados (Fassbender \& Bornemisza, 1987; Olsen \& Khasawneh, 1980).

La capacidad del suelo para retener cationes y aniones solubles depende en gran medida del coloide y de la carga del ión. La retención aniónica tiene gran importancia tanto en los suelos tropicales como en aquellos fuertemente intemperizados. Estos poseen óxidos hidratados de aluminio $\left(\mathrm{Al}^{+3}\right)$ y de hierro $\left(\mathrm{Fe}^{+3}\right)$ cuya carga negativa es considerablemente menor que la positiva, sobre todo a $\mathrm{pH}$ bajo, y se denominan suelos de carga variable debido a que retienen con mas facilidad los aniones que los cationes (Fassbender \& Bornemisza, 1987).

Según Mejía (1996), la carga variable de los oxisoles y suelos óxicos proviene de los óxidos e hidróxidos de $\mathrm{Fe}^{+3}$ y $\mathrm{Al}^{+3}$ que se comportan como sustancias anfóteras, dependiendo del pH. Así, la adición de cal agrícola y fosfatos, induce un incremento en la carga neta negativa de los oxisoles, incrementa el $\mathrm{pH}$, mientras que los fosfatos al disminuir el $\mathrm{pH}$ del punto isoeléctrico aumentan la CIC en $0,8 \mathrm{cmol}$, por cada mmol de $\mathrm{P}$ adsorbido.

No obstante, Berkowitz et al. (2005) mostraron que al incrementar la concentración de aluminio, la cristalinidad del hidróxido de aluminio aumenta, induciendo la transformación del $\mathrm{Al}(\mathrm{OH})_{3}$ amorfo a gibsita. Esta transformación disminuye el área superficial así como la adsorción de fosfato medida mediante isotermas de adsorción (Berkowitz et al., 2006), lo que incrementa la concentración de $\mathrm{Al}^{+3}$ en el suelo y consecuentemente, problemas de toxicidad en las plantas.

La materia orgánica favorece la disponibilidad del fósforo en los oxisoles ya que contribuye a disminuir la retención de fosfatos. Guggenberger et al. (1996) encontraron baja retención de $\mathrm{P}$ inorgánico en oxisoles y este hecho lo relacionaron con el contenido de materia orgánica que se encontraba en la capa superficial. La afinidad del suelo por el P inorgánico disminuyó significativamente con el establecimiento de pastos, llegando a presentarse la más alta concentración de $\mathrm{P}$ en equilibrio en los desechos de lombrices que se encontraban en este sistema de producción.

Así mismo Borggaard et al. (2005) encontraron que las concentraciones de sustancias húmicas provenientes de la materia orgánica, que normalmente se encuentran en los podzoles y umbrisoles, no afectan la adsorción del fósforo ocasionada por la presencia de óxidos de hierro y aluminio.

Adiciones previas de $\mathrm{P}$ reducen la capacidad de los suelos para adsorber más fosfatos. Barrow (1974) incubó cuatro suelos por 12 meses con varios niveles de fosfatos y luego determinó las isotermas comparándolos con un testigo inicial, encontrando que las curvas de las isotermas de los suelos incubados no solo se habían desplazado hacia la derecha con respecto al suelo inicial, sino que también la pendiente de las curvas era menor, lo que significa que la capacidad buffer del fosfato disminuyó, lo que indica que hubo una conversión del fosfato a formas que ocuparon sitios de adsorción. Bajos niveles de aplicación produjeron mayor efecto que los altos niveles.

Del mismo modo Hunter et al. (1997) al hacer aplicaciones de residuos de cosecha a un oxisol encontraron que la disponibilidad del $\mathrm{P}$ en el suelo fue mayor, y la producción de granos de maíz se incrementó. En general, la fertilización con materiales orgánicos (desechos de cosecha y de animales, entre otros) constituyen una buena enmienda para el suelo, ya que incrementan la fertilidad del suelo y reducen la acidez del mismo por unos pocos meses (Hue \& Amien, 1989).

De acuerdo a lo anterior, en este estudio se adicionaron diferentes enmiendas tanto orgánicas como inorgánicas a un oxisol con el objetivo de determinar a través de las isotermas de Langmuir el grado de adsorción del fósforo, para establecer disponibilidad para las plantas.

\section{MATERIAL Y MÉTODOS}

El suelo en estudio presentó una textura Franco Arcillo Arenosa y esta clasificado como Inceptic Hapludox. Las muestras fueron tomadas en el Centro de Investigaciones Corpoica-La Libertad, ubicado en el Piedemonte Andino (Villavicencio - Colombia), con coordenadas $9^{\circ} 6^{\prime}$ de latitud norte, $73^{\circ} 34^{\prime}$ de longitud y $320 \mathrm{msnm}$, con una temperatura media de $27^{\circ} \mathrm{C}$ y humedad relativa entre el 70 y el $90 \%$. Las muestras fueron tomadas a una profundidad $0,20 \mathrm{~m}$. En la Tabla 1 se encuentran las principales propiedades físicas y químicas del suelo estudiado.

Se utilizaron dos abonos orgánicos, el primero de origen animal, gallinaza secada al aire proveniente de los galpones de gallinas ponedoras, con 15 semanas de compostaje (incu-

Tabla 1. Características físicas y químicas del suelo en estudio

\begin{tabular}{lcc}
\hline \multicolumn{1}{c}{ Característica } & Unidad & Valor \\
\hline $\mathrm{MO}$ & $\%$ & 2,96 \\
$\mathrm{pH}$ & & 4,7 \\
$\mathrm{P}$ Disponible & $\mathrm{mg} \mathrm{kg}^{-1}$ & 69 \\
Total & $\mathrm{cmol}_{\mathrm{c} \mathrm{kg}}$ & 65 \\
$\mathrm{Al}+\mathrm{H}$ & $\mathrm{Cmol}_{\mathrm{c}} \mathrm{kg}^{-1}$ & 2,2 \\
$\mathrm{Al}$ & $\mathrm{Cmol}_{\mathrm{c}} \mathrm{kg}^{-1}$ & 2,0 \\
$\mathrm{Ca}$ & $\mathrm{Cmol}_{\mathrm{c}} \mathrm{kg}^{-1}$ & 0,12 \\
$\mathrm{Mg}$ & $\mathrm{Cmol}_{\mathrm{c}} \mathrm{kg}^{-1}$ & 0,07 \\
$\mathrm{~K}$ & $\mathrm{Cmol}_{\mathrm{c}} \mathrm{kg}^{-1}$ & 0,05 \\
$\mathrm{Na}$ & $\mathrm{cmol}_{\mathrm{c} \mathrm{kg}}$ & 0,07 \\
$\mathrm{CIC}$ & $\mathrm{mg} \mathrm{kg}^{-1}$ & 4,51 \\
$\mathrm{~S}$ & $\mathrm{mg} \mathrm{kg}^{-1}$ & 6 \\
$\mathrm{~B}$ & $\mathrm{mg} \mathrm{kg}^{-1}$ & 0,0 \\
$\mathrm{Zn}$ & $\mathrm{mg} \mathrm{kg}^{-1}$ & 5 \\
$\mathrm{Mn}$ & $\mathrm{mg} \mathrm{kg}^{-1}$ & 2,4 \\
$\mathrm{Cu}$ & $\mathrm{mg} \mathrm{kg}^{-1}$ & 1,1 \\
$\mathrm{Fe}$ & & 14,0 \\
\hline
\end{tabular}


bación) y el segundo de origen vegetal, compost proveniente de residuos de flores (tallos y raíces) con un tiempo de incubación de 10 semanas. Las principales características de los materiales orgánicos utilizados se presentan en la Tabla 2. Como planta indicadora se sembró en las bolsas maíz (Zea mays L.) híbrido master, resistente al aluminio. Así mismo se utilizó un abono químico en una cantidad de $0,0089 \mathrm{~g}$ de $\mathrm{P}_{2} \mathrm{O}_{5}$ por cada $100 \mathrm{~g}$ de suelo $(0.019 \mathrm{~g}$ de SPT por cada 100 $\mathrm{g}$ de suelo), con el fin de incrementar la cantidad de $\mathrm{P}$ total en el suelo y estimar las isotermas de adsorción, así como la eficiencia del uso de los dos tipos de enmienda.

Tabla 2. Características de los materiales orgánicos

\begin{tabular}{lccc}
\hline \multicolumn{1}{c}{ Característica } & Unidad & Compost & Gallinaza \\
\hline $\mathrm{pH}$ & & 7,0 & 8,7 \\
Humedad & $\%$ & 34,0 & 35,0 \\
$\mathrm{MO}$ & $\%$ & 62,0 & 63,0 \\
$\mathrm{C}$ & $\%$ & 12,7 & 12,3 \\
$\mathrm{~N}$ & $\%$ & 34,0 & 35,0 \\
$\mathrm{CaCO}{ }_{3}$ & $\%$ & 32,0 & 34,0 \\
$\mathrm{P}$ & $\%$ & 0,7 & 6,0 \\
$\mathrm{~K}$ & $\%$ & 2,6 & 4,6 \\
$\mathrm{Mg}$ & $\%$ & 0,8 & 2,2 \\
$\mathrm{Ca}$ & $\%$ & 11,0 & 10,0 \\
$\mathrm{Na}$ & $\%$ & 0,5 & 0,4 \\
$\mathrm{~S}$ & $\%$ & 1,0 & 0,5 \\
$\mathrm{~B}$ & $\mathrm{mg} \mathrm{kg}-1$ & 72 & 54 \\
$\mathrm{Fe}$ & $\mathrm{mg} \mathrm{kg}$ & 668 & 6560 \\
$\mathrm{Cu}$ & $\mathrm{mg} \mathrm{kg}^{-1}$ & 63 & 180 \\
$\mathrm{Zn}$ & $\mathrm{mg} \mathrm{kg}$ & 2148 \\
$\mathrm{Mn}$ & $\mathrm{mg} \mathrm{kg}$ & 254 & 752 \\
$\mathrm{C} / \mathrm{N}$ & $\mathrm{mg} \mathrm{kg}^{-1}$ & 642 & 12,3 \\
\hline
\end{tabular}

Debido a la acidez de este tipo de suelos además de los anteriores fertilizantes, se utilizó cal agrícola como enmien$\mathrm{da}$, con el fin de elevar el $\mathrm{pH}$ del suelo y favorecer el aumento de la concentración de iones en la solución del suelo y su disponibilidad para las plantas.

El suelo a estudiar fue secado al aire y tamizado por una malla de $4 \mathrm{~mm}$, colocado en bolsas negras de polietileno de acuerdo con los tratamientos que aparecen en la Tabla 3, los cuales consistieron en la mezcla del suelo tanto con los abonos orgánicos como con el fertilizante químico y la cal agrí- cola, dando lugar a 13 tratamientos con tres repeticiones cada uno para un total de 39 unidades experimentales que se dispusieron en bloques al azar, bajo invernadero.

El estudio de adsorción del fósforo se realizó en muestras de suelo en el momento de la siembra para determinar las condiciones iniciales del experimento y en la semana 10, ya que durante este tiempo ocurre la máxima absorción de fósforo por la planta. Para ello se colocaron $2,5 \mathrm{~g}$ de suelo con $25 \mathrm{ml}$ de $\mathrm{CaCl}_{2} 0,01 \mathrm{M}$ que contiene $\mathrm{KH}_{2} \mathrm{PO}_{4}$ en las concentraciones de $0 ; 2,5 ; 5,0 ; 10,0 ; 20,0 ; 50,0 ; 75,0100$ y 200 $\mathrm{mg} \mathrm{kg}{ }^{-1}$ de P por 5 días de acuerdo a Fox \& Kamprath (1970). Las muestras se agitaron por 16 horas todos los días. Después del periodo de equilibrio las muestras se centrifugaron a $3500 \mathrm{rpm}$ por 15 minutos. Se tomó una alícuota del sobrenadante para determinar la concentración de $\mathrm{P}$ colorimétricamente por el método del molibdato de amonio (Mota et al., 1990; Murphy \& Riley, 1962). El P adsorbido se determinó por diferencia entre el $\mathrm{P}$ adicionado inicialmente y el $\mathrm{P}$ remanente en la solución después del periodo de equilibrio.

Se utilizaron las isotermas de adsorción, obteniendo curvas de la concentración de $\mathrm{P}$ en equilibrio en la solución del suelo contra el $\mathrm{P}$ adsorbido para los distintos tratamientos, ya que estas describen la fuerza con que el soluto es adsorbido por un sólido a temperatura y presión constante, y muestran la cantidad de adsorbato en función de su concentración en equilibrio (Bohn et al., 1993). Los datos obtenidos se ajustaron al modelo propuesto por Langmuir ya que estas ecuaciones han sido las más utilizadas para caracterizar la adsorción de P en los suelos (Porta et al., 1994; Zhou et al., 2005; Das et al., 2006).

La ecuación de Langmuir está dada por:

$$
q=\frac{b \cdot k \cdot c}{(1+k \cdot c)}
$$

donde $q$ es la cantidad de $\mathrm{P}$ adsorbido por unidad de masa de suelo ( $\left.\mathrm{mol} \mathrm{g}^{-1}\right) ; b$ es la adsorción máxima de $\mathrm{P}\left(\mathrm{mg} \mathrm{g}^{-1}\right)$; $c$ es la concentración de $\mathrm{P}$ en equilibrio en la solución (mol $\mathrm{L}^{-1}$ ); y $k$ es una constante relacionada con la energía de enlace (Liu, 2006). Esta ecuación es utilizada en rangos limitados de concentración de $\mathrm{P}\left(<0,10 \mathrm{mg} \mathrm{g}^{-1}\right)$. Bornemisza

Tabla 3. Descripción de los tratamientos

\begin{tabular}{|c|c|c|c|c|c|c|c|}
\hline \multirow{2}{*}{ Tratamiento } & Gallinaza & Compost & Cal & SPT & MO & $\mathrm{CaCO}_{3}$ & $\mathbf{P}_{2} \mathbf{O}_{5}$ \\
\hline & \multicolumn{3}{|c|}{ Cantidad de material $\mathrm{g} / 100 \mathrm{~g}$ de suelo } & \multicolumn{4}{|c|}{ Aportes $\mathrm{g} / \mathrm{l00 \textrm {g }}$ de suelo } \\
\hline T1 (Testigo) & 0 & 0 & 0 & 0 & 0 & 0 & 0 \\
\hline T2 (Gallinaza) & 1,28 & 0 & 0 & 0 & 0,8 & 0,44 & 0,16 \\
\hline T3 (Compost) & 0 & 1,28 & 0 & 0 & 0,8 & 0,40 & 0,021 \\
\hline T4 (Compost + cal*) & 0 & 1,28 & 0,07 & 0 & 0,8 & 0,44 & 0,021 \\
\hline T5 (Compost + cal**) & 0 & 1,28 & 0,16 & 0 & 0,8 & 0,48 & 0,021 \\
\hline T6 (Fertilizante químico) & 0 & 0 & 0 & 0,019 & 0 & 0 & 0,0089 \\
\hline T7 (Fertilizante químico + cal*) & 0 & 0 & 0,81 & 0,019 & & 0,44 & 0,0089 \\
\hline T8 (Fertilizante químico + cal**) & 0 & 0 & 0,16 & 0,019 & 0 & 0,084 & 0,0089 \\
\hline T9 (Cal*) & 0 & 0 & 0,81 & 0 & 0 & 0,44 & 0 \\
\hline $\mathrm{T} 10\left(\left.\mathrm{Ca}\right|^{* *}\right)$ & 0 & 0 & 0,16 & 0 & 0 & 0,084 & 0 \\
\hline T11 (Gallinaza + Fertilizante químico) & 0,64 & 0 & 0 & 0,019 & 0,4 & 0,22 & 0,17 \\
\hline T12 (Compost + Fertilizante químico) & 0 & 0,64 & 0 & 0,019 & 0,4 & 0,20 & 0,03 \\
\hline T13 (Compost + Fertilizante químico + cal***) & 0 & 0,64 & 0,07 & 0,019 & 0,8 & 0,24 & 0,03 \\
\hline
\end{tabular}

* 12,3 $\mathrm{Mg} \mathrm{ha}^{-1} \mathrm{de} \mathrm{Cal} ; * * 2,0 \mathrm{Mg} \mathrm{ha}^{-1} \mathrm{de} \mathrm{Cal} ; * * * 0,9 \mathrm{Mg} \mathrm{ha}^{-1} \mathrm{de} \mathrm{Cal}$ 
(1982) también asume que la adsorción del ión fósforo se da en monocapa sobre la superficie del mineral, lo cual ocurre cuando se tiene altas concentraciones de $\mathrm{P}$ en la solución del suelo (Havlin et al., 1999).

Mediante esta ecuación se puede observar el mecanismo responsable de la adsorción de $\mathrm{P}$ (óxidos o hidróxidos de $\mathrm{Fe}$ y Al, arcillas caolinitas o carbonatos), por lo que son interesantes para conocer si el $\mathrm{P}$ no lábil puede llegar a ser disponible para las plantas.

El análisis estadístico se realizó por medio de un análisis de varianza con una confiabilidad del $95 \%$. Se utilizó la prueba de comparación de medias de Tukey. El paquete estadístico utilizado para el análisis de los datos fue el SAS v. 8.1e (Cary, N.C)

\section{RESULTADOS Y DISCUSIÓN}

Con el fin de determinar si la adsorción de P sigue la isoterma de Langmuir, es decir si hay una adsorción máxima, se determinaron las curvas a partir de los valores de la concentración de $\mathrm{P}$ en la solución de equilibrio (Ceq, $\mathrm{mg} \mathrm{L}^{-1}$ ) contra la cantidad de $\mathrm{P}$ adsorbido por gramo de suelo (q, $\mathrm{mg} \mathrm{g}^{-1}$ ). Todos los tratamientos mostraron la misma tendencia en la curva y alcanzaron una adsorción máxima acorde a lo encontrado por Porta et al. (1994). La cantidad de P adsorbida en cada tratamiento aumentó con el contenido de P en la solución del suelo. A concentraciones más altas, las curvas se volvieron asintóticas y se acotaron a un máximo, lo cual indica la existencia de una quimiadsorción.

Al comparar el efecto de las enmiendas por sí solas, tratamientos T1, T2, T3, T6 y T9 $\left(0,0081 \mathrm{~g} \mathrm{cal} \mathrm{g}^{-1}\right.$ suelo) en las Figuras 1A y 1B, se observa que el fertilizante químico proporciona menor adsorción máxima, no obstante cabe resaltar que al aplicar los fertilizante orgánicos la adsorción también disminuyó, lo que indica que las enmiendas orgánicas e inorgánicas inhibieron al aluminio y limitaron la fijación de P.

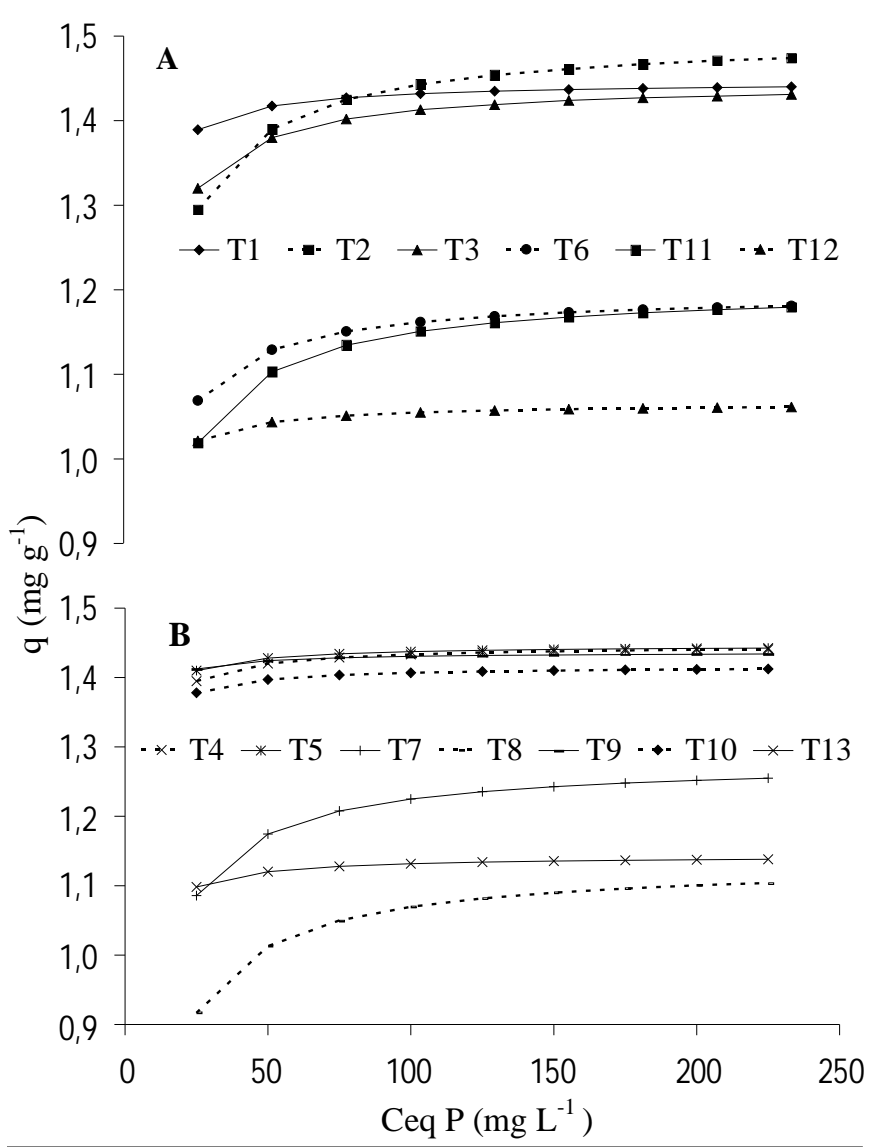

Figura 1. Isotermas de adsorción de $\mathrm{P}$ al momento de la siembra (A y B) para los diferentes tratamientos. T1: testigo; T2: gallinaza; T3: compost; T4: compost + cal $12,3 \mathrm{Mg} \mathrm{ha}^{-1}$; T5: compost + cal 2,0 $\mathrm{Mg} \mathrm{ha}^{-1}$; T6: fertilizante químico; T7: fertilizante químico + cal $12,3 \mathrm{M} \mathrm{g} \mathrm{ha}^{-1} ;$; 8 : fertilizante químico + cal $2,0 \mathrm{M} \mathrm{g} \mathrm{ha}^{-1}$;

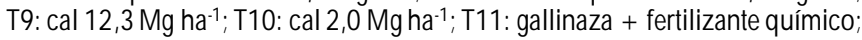
T12: compost + fertilizante químico; T13: compost + fertilizante químico + cal $0,9 \mathrm{M} \mathrm{g} \mathrm{ha}^{-1}$

Cuando se utilizó gallinaza, disminuyó la adsorción de $\mathrm{P}$ al adicionar concentraciones menores de $25 \mathrm{mg} \mathrm{L}^{-1}$ de $\mathrm{P}$ y aumentó la retención con concentraciones mayores a los 75 $\mathrm{mg} \mathrm{L}^{-1}$ de P. La alta adición de $\mathrm{P}$ fue insuficiente para disminuir la adsorción; sin embargo, no se pudo determinar el

Tabla 4. Expresión lineal de la ecuación de Langmuir, capacidad máxima de adsorción (mg g-1 de suelo) y constante de energía de adsorción de Pal momento de la siembra para todos los tratamientos

\begin{tabular}{|c|c|c|c|c|}
\hline Tratamiento & Ecuación & $\mathbf{R}$ & CMA & $\bar{K}$ \\
\hline T1 (Testigo) & $Y=0,6542 x+0,5765$ & 0,982 & $1,40 a b$ & $0,93 a b c$ \\
\hline T2 (Gallinaza) & $Y=0,6868 x+1,9073$ & 0,943 & $1,45 \mathrm{a}$ & $0,24 d$ \\
\hline T3 (Compost) & $Y=0,6523 x+1,0306$ & 0,972 & $1,40 a b$ & $0,40 \mathrm{~cd}$ \\
\hline T4 (Compost + cal*) & $Y=0,6480 x+0,4268$ & 0,991 & $1,40 a b$ & 1,04 bcd \\
\hline T5 (Compost + cal**) & $Y=0,6538 x+0,3107$ & 0,993 & $1,40 a b$ & $1,48 a b$ \\
\hline T6 (Fertilizante químico) & $Y=0,7945 x+1,6838$ & 0,958 & 1,15 bcde & $0,32 \mathrm{~cd}$ \\
\hline T7 (Fertilizante químico + cal*) & $Y=0,7698 x+3,3623$ & 0,888 & 1,23 abcde & $0,21 d$ \\
\hline T8 (Fertilizante químico + cal**) & $Y=0,9653 x+5,8347$ & 0,852 & $1,08 \mathrm{e}$ & $0,16 d$ \\
\hline T9 $\left.(\mathrm{Ca})^{*}\right)$ & $Y=0,6875 x+0,3522$ & 0,998 & $1,39 a b$ & $2,23 a$ \\
\hline T10 (Cal**) & $Y=0,7886 x+0,5325$ & 0,999 & $1,37 \mathrm{abcd}$ & $1,38 a b c$ \\
\hline T11 (Gallinaza + Fertilizante químico) & $Y=0,9272 x+4,9721$ & 0,900 & 1,15 abcde & $0,21 d$ \\
\hline T12 (Compost + Fertilizante químico) & $Y=0,8978 x+0,6890$ & 0,999 & $1,08 \mathrm{e}$ & $0,86 \mathrm{bcd}$ \\
\hline T13 (Compost+ Fertilizante químico + cal***) & $Y=0,9962 x+0,6991$ & 0,999 & 1,09 de & $0,93 \mathrm{bcd}$ \\
\hline
\end{tabular}

Y: concentración de $\mathrm{P}$ adsorbido ( $\mathrm{mg} \mathrm{g}^{-1}$ de suelo); x: concentración de $\mathrm{P}$ en equilibrio (Ceq $\mathrm{mg} \mathrm{L}^{-1}$ ); R: coeficiente de correlación; K: constante de energía de adsorción de P; CMA: capacidad máxima de adsorción; *:12,3 $\mathrm{Mg} \mathrm{ha}^{-1} \mathrm{de}$ cal; **:2,0 $\mathrm{Mg} \mathrm{ha}^{-1} \mathrm{de}$ cal; ***:0,9 $\mathrm{Mg} \mathrm{ha}^{-1}$ de cal; valores seguidos de letras distintas en cada columna indican diferencia significativa, según prueba de Tukey $(\mathrm{P}<0,05)$ 
tipo de adsorción que ocurrió, ya que en el tiempo se siguió conservando la tendencia y el valor permaneció inalterado. Además, se debe considerar que en el suelo hay presencia de microorganismos que aumentan su actividad con la temperatura lo que conlleva a incrementar los procesos de inmovilización del P. Para este estudio la temperatura media del suelo fue $28^{\circ} \mathrm{C}$ a $0,50 \mathrm{~m}$ de profundidad, siendo superior cerca de la superficie, lo cual promueve la actividad microbiana.

Tanto el testigo como los tratamientos con cal agrícola y compost tuvieron un comportamiento similar; valores altos de las constantes de energía y de la capacidad máxima de retención (Tabla 4), indicando que bajos contenidos de $\mathrm{P}$ en un suelo con $\mathrm{pH}$ ácido no garantizan un adecuado suministro a las plantas, dado que el elemento en su totalidad fue fijado por los minerales del suelo.

También se encontró que al utilizar el fertilizante fosfórico por si solo, se mantuvo una menor capacidad de retención de $\mathrm{P}$ que cuando se mezcló con $12,3 \mathrm{Mg} \mathrm{ha}^{-1}$ de Cal (Tabla 4). Sin embargo no se encontraron diferencias significativas entre los tratamientos. Esto supone que para disminuir la capacidad de retención, no basta con subir el $\mathrm{pH}$ y neutralizar la cantidad de aluminio intercambiable, sino que influye de gran manera el suministro de P. Se encontró que el uso excesivo de cal no es tan eficiente para disminuir la cantidad de $\mathrm{P}$ adsorbido, aunque esto puede deberse a que la cal en dosis altas necesita un mayor tiempo para solubilizarse y así ejercer su efecto.

Respecto a los valores de adsorción máxima, cuando se adicionaron concentraciones menores o iguales a $25 \mathrm{mg} \mathrm{L}^{-1}$, el suelo adsorbió la totalidad del $\mathrm{P}$ en todos los tratamientos, mostrando que a estas concentraciones el suelo fija $\mathrm{P}$ independientemente de si su origen es mineral u orgánico (Figura 1A y 1B). Esta situación también puede estar originada en el hecho de que a valores de $\mathrm{pH}$ en el suelo cercanos a 5, se presenta la máxima adsorción de P (Das et al., 2006). No obstante, al adicionar enmiendas con cal y fósforo en los tratamientos, el $\mathrm{pH}$ se incrementa y consecuentemente hace que la adsorción del fosfato disminuya.
Fox \& Kamprath (1970) mostraron que en los oxisoles la adsorción máxima de $\mathrm{P}$ estuvo entre 0,50 y $0,70 \mathrm{mg} \mathrm{g}^{-1}$ y que requería de hasta $0,90 \mathrm{mg} \mathrm{g}^{-1}$ de $\mathrm{P}$ para alcanzar un nivel adecuado $\left(0,2 \mathrm{mg} \mathrm{kg}^{-1}\right)$ en la solución del suelo. Valores que resultan ser más bajos que los encontrados en este estudio (entre 1,10 y $1,50 \mathrm{mg} \mathrm{g}^{-1}$ ), son debidos posiblemente a la presencia de hidróxidos de $\mathrm{Al}-\mathrm{Mg}$ de doble capa difusa, que de acuerdo con Das et al. (2006) realizan una mayor adsorción del fósforo presente en la solución del suelo. Por otro lado estos valores distan de lo encontrado por Cepeda \& Narro (1986) en un suelo calcáreo en donde la adsorción máxima varía entre 0,002 y $0,03 \mathrm{mg}$ $\mathrm{g}^{-1}$, y por Zhou et al. (2005) en los sedimentos de un lago $\left(0,05\right.$ a $\left.0,30 \mathrm{mg} \mathrm{g}^{-1}\right)$.

Benavides (1978) estudió la adsorción de P en nueve suelos de la Amazonía colombiana, utilizando las isotermas de Langmuir y observó que para obtener valores de intensidad en la solución del suelo de 0,2 y $0,3 \mathrm{mg} \mathrm{L}^{-1}$ se necesitaron cantidades de P entre 0,091 y $0,312 \mathrm{mg}$ $\mathrm{g}^{-1}$, equivalentes al $17 \%$ y $40 \%$ de la adsorción máxima, y entre 0,095 y $0,370 \mathrm{mg} \mathrm{g}^{-1}$, equivalentes al 18 y $49 \%$ respectivamente. Del mismo modo, para suelos con horizontes óxicos encontró una adsorción máxima entre 0,82 y $0,87 \mathrm{mg} \mathrm{g}^{-1}$ y constantes de energías de adsorción entre 1,8 y 2,5 .

Fassbender (1966) estudió la adsorción de fosfatos en tres tipos de suelos, entre ellos los latosoles, utilizando la ecuación de Langmuir y obtuvo capacidades máximas de adsorción comprendidas entre 0,50 y $2,0 \mathrm{mg} \mathrm{g}^{-1}$ de $\mathrm{P}$ adsorbido y constantes de energía de adsorción entre 0,15 y 0,29. Arias et al. (2006) observaron que en el cuarzo, los hidróxidos de hierro y aluminio presentes pueden llegar a retener $\mathrm{P}$ a valores máximos de 4,0 y $6,0 \mathrm{mg} \mathrm{g}^{-1}$ respectivamente.

Al comparar las isotermas de adsorción de $\mathrm{P}$ al momento de siembra con las obtenidas diez semanas después (Figura 1A, 1B, 2A y 2B), se observa que en todos los tratamientos la adsorción de $\mathrm{P}$ se incrementó nuevamente a los valores cercanos al testigo, lo que indica que

Tabla 5. Expresión lineal de la ecuación de Langmuir, capacidad máxima de adsorción ( $\mathrm{mg} \mathrm{g}^{-1}$ de suelo) y constante de energía de adsorción de P, diez semanas después de la siembra para todos los tratamientos

\begin{tabular}{|c|c|c|c|c|}
\hline Tratamiento & Ecuación & $\mathbf{R}$ & CMA & $\mathrm{K}$ \\
\hline T1 (Testigo) & $Y=0,6726 x+0,5684$ & 0,990 & $1,45 a b c$ & $3,56 a$ \\
\hline T2 (Gallinaza) & $Y=0,7417 x+0,4645$ & 0,997 & $1,41 a b c$ & $2,91 \mathrm{a}$ \\
\hline T3 (Compost) & $Y=0,7280 x+0,2413$ & 0,998 & $1,49 a$ & $2,78 \mathrm{a}$ \\
\hline T4 (Compost + cal*) & $Y=0,7302 x+0,4508$ & 0,991 & $1,43 a b c$ & $1,46 a$ \\
\hline T5 (Compost + cal**) & $Y=0,7251 x+0,2006$ & 0,999 & $1,33 a b c$ & $2,48 a$ \\
\hline T6 (Fertilizante químico) & $Y=0,7757 x+0,3664$ & 0,957 & $1,25 \mathrm{C}$ & $1,56 \mathrm{a}$ \\
\hline T7 (Fertilizante químico + cal*) & $Y=0,8070 x+0,2687$ & 0,999 & $1,40 a b c$ & $2,09 a$ \\
\hline T8 (Fertilizante químico + cal**) & $Y=0,8134 x+0,2703$ & 0,999 & $1,35 a b c$ & $2,05 a$ \\
\hline T9 (Cal*) & $Y=0,7203 x+0,1823$ & 0,999 & $1,39 a b c$ & $2,69 a$ \\
\hline T10 (Cal**) & $Y=0,7513 x+0,1338$ & 0,999 & $1,46 a b$ & $3,63 a$ \\
\hline T11 (Gallinaza + Fertilizante químico) & $Y=0,7970 x+0,5376$ & 0,997 & $1,27 b c$ & $1,07 a$ \\
\hline T12 (Compost + Fertilizante químico) & $Y=0,7970 x+0,5156$ & 0,998 & $1,29 b c$ & $1,07 \mathrm{a}$ \\
\hline T13 (Compost + Fertilizante químico + cal***) & $Y=0,7412 x+0,6105$ & 0,990 & $1,39 a b c$ & $0,88 \mathrm{a}$ \\
\hline
\end{tabular}

Y: concentración de $\mathrm{P}$ adsorbido ( $\mathrm{mg} \mathrm{g}^{-1}$ de suelo); $\mathrm{x}$ : concentración de $\mathrm{P}$ en equilibrio (Ceq mg $\mathrm{L}^{-1}$ ); R: coeficiente de correlación; K: constante de energía de adsorción de P; CMA: capacidad máxima de adsorción; *:12,3 $\mathrm{Mg} \mathrm{ha}^{-1}$ de cal; **:2,0 $\mathrm{Mg} \mathrm{ha}^{-1}$ de cal; ***:0,9 Mg ha-1 de cal; valores seguidos de letras distintas en cada columna indican diferencia significativa, según prueba de Tukey $(\mathrm{P}<0,05)$ 


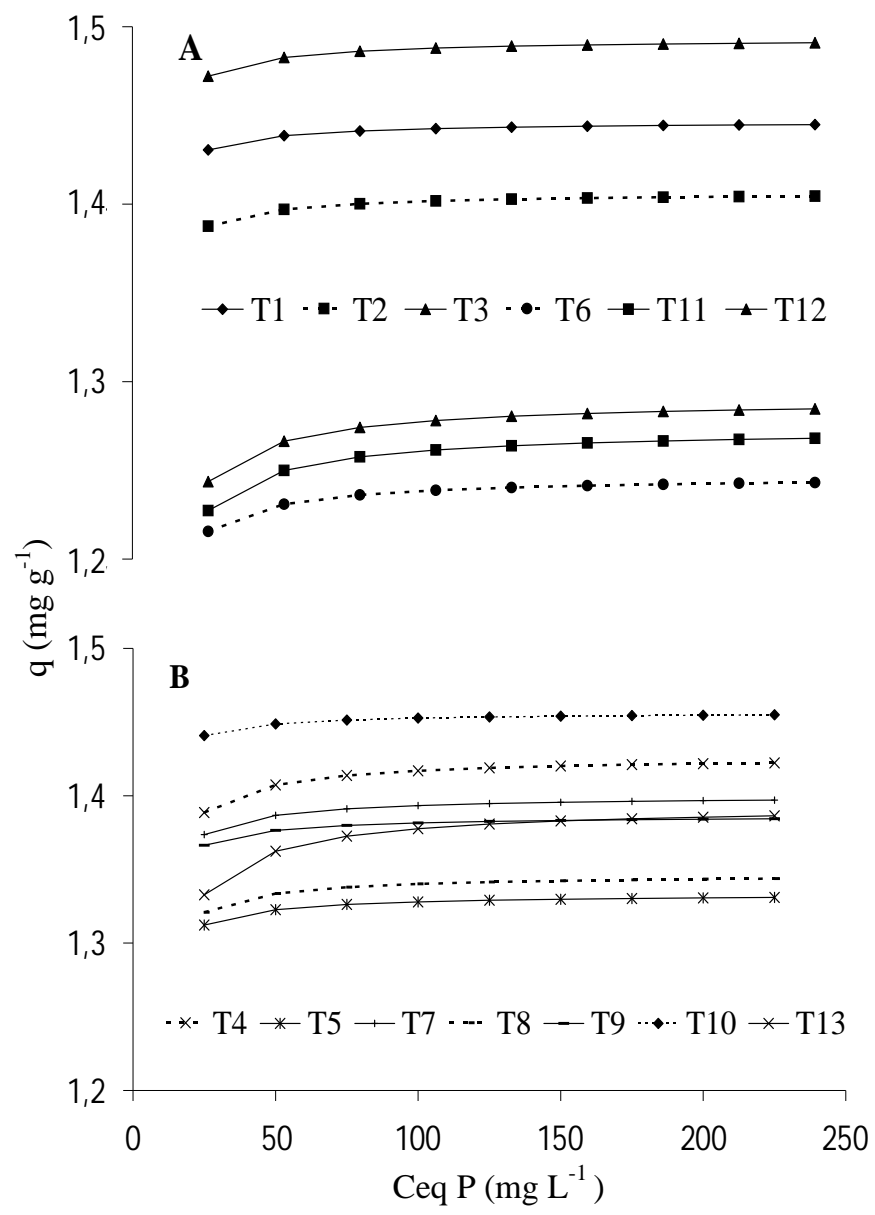

Figura 2. Isotermas de adsorción de $\mathrm{P}$ diez días después de la siembra (A y $\mathrm{B}$ ) para los diferentes tratamientos. T1: testigo; T2: gallinaza; T3: compost; T4: compost + cal 12,3 $\mathrm{M} \mathrm{g} \mathrm{ha}^{-1} ;$ T5: compost + cal 2,0 M g ha-1; T6: fertilizante químico; T7: fertilizante químico + cal $12,3 \mathrm{M} \mathrm{g} \mathrm{ha}^{-1}$; T8: fertilizante químico

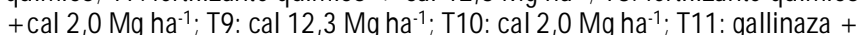
fertilizante químico; T12: compost + fertilizante químico; T13: compost + fertilizante químico + cal 0,9 $\mathrm{Mg} \mathrm{ha}^{-1}$

tanto la adición de enmiendas orgánicas como inorgánicas es una solución temporal a la fijación de $\mathrm{P}$ y se requiere la aplicación de enmiendas químicas y orgánicas periódicamente para incrementar la cantidad de $\mathrm{P}$ en solución y su respectiva disponibilidad para las plantas. Esto puede ocurrir debido a que las zonas en donde se presentan este tipo de suelos (oxisoles y ultisoles) son zonas con alta intemperización, originando una mineralización muy rápida de la materia orgánica y una lixivación de los fertilizantes aplicados, limitando la duración del efecto de la adición de estos materiales sobre la adsorción de P.

Las Tablas 4 y 5 se presentan las expresiones lineales de la ecuación de Langmuir, la adsorción máxima determinada a partir de la pendiente de la curva, las constantes de energía o afinidad y los coeficientes de correlación para los tratamientos estudiados en las dos épocas analizadas. Las curvas presentan un coeficiente de correlación cercano a uno y positivo, lo que indica que a mayor concentración de $\mathrm{P}$ en equilibrio, mayor será el $\mathrm{P}$ adsorbido. Polyzopoulos et al. (1985) estudiaron 14 suelos del orden alfisol en Grecia, utilizando la ecuación de
Langmuir y Freundlich y obtuvieron que independientemente del modelo, las curvas obtenidas no tienen forma rectilínea sino que presentan cierta curvatura generada posiblemente porque los coloides del suelo no presentan sitios de adsorción homogéneos, contrario a los supuestos de los modelos de isotermas.

Las isotermas examinadas son una aproximación adecuada para describir la adsorción de $\mathrm{P}$ en los oxisoles. Estas permiten conocer la disponibilidad de $\mathrm{P}$ en el suelo de acuerdo a la concentración de P en la solución. Del mismo modo, brindan la posibilidad de conocer si el $\mathrm{P}$ no lábil puede pasar a la solución del suelo y estar disponible para las plantas. Según Havlin et al. (1999) las isotermas no permiten determinar el mecanismo responsable de la adsorción (óxidos o hidróxidos de $\mathrm{Fe}$ y Al, arcillas caolinitas o carbonatos), y tampoco tienen en cuenta el hecho de que los sitios de adsorción no son energéticamente uniformes, los iones fosfato interactúan lateralmente y afectan la actividad superficial de las arcillas, la carga negativa se incrementa continuamente con la adsorción de P, y que la solución del suelo cambia constantemente.

En resumen, para el suelo estudiado, el cual posee bajos contenidos de $\mathrm{P}$, no solo se necesitó aplicar una enmienda que facilitara la liberación del P ligado ya fuese a los óxidos e hidróxidos de hierro y aluminio, sino que también fue necesario adicionar el elemento con el fin de garantizar el aporte del nutrimento a la planta. Así mismo, se encontró que al combinar los fertilizantes químicos y orgánicos se generó la menor retención de fosfatos, y presentaron constantes de afinidad medias, incrementando la concentración de $\mathrm{P}$ en la solución del suelo y mejorando el suministro a la planta.

\section{CONCLUSIONES}

1. En el momento de la siembra, los tratamientos que combinaron fertilizantes orgánicos y químicos fueron los que mejor suministraron $\mathrm{P}$ al suelo y por tanto a las plantas.

2. Para disminuir la capacidad de retención de $\mathrm{P}$ de un suelo, no basta con subir el $\mathrm{pH}$ y disminuir el porcentaje de aluminio intercambiable, sino que es necesario el suministro de $P$.

3. El tratamiento que resultó más eficiente en el momento de la siembra para disminuir la fijación de P por óxidos e hidróxidos de aluminio y hierro fue la combinación de la gallinaza con el fertilizante fosfórico, que puede ocurrir debido tanto al contenido de materia orgánica, como al de carbonato de calcio $\left(\mathrm{CaCO}_{3}\right)$ y al elevado contenido de P.

4. Se encontró que al combinar los fertilizantes químicos y orgánicos se obtuvo la menor retención de fosfatos, y constantes de afinidad medias lo que garantiza el suministro del nutriente a la planta.

5. El efecto de las enmiendas estudiadas es temporal pues al cabo de 10 semanas la adsorción del P por los coloides se da en la misma intensidad que en el testigo, por lo que se deben 
realizar aplicaciones periódicas con el fin de mantener una concentración adecuada de $\mathrm{P}$ en la solución del suelo.

\section{LITERATURA CITADA}

Arias, M.; Silva-Carballal, J. da; Garcia-Rio, L.; Mejuto, J.; Nunez, A. Retention of phosphorus by iron and aluminum-oxides-coated quartz particles. Journal of Colloid and Interface Science, v.295, n.1, p.65-70, 2006.

Barrow, N. Effect of previous additions of phosphate on phosphate adsorption by soils. Soil Science, v.118, n.2, p.82-89, 1974.

Benavides, G. de. Adsorción del fósforo en suelos de la Amazonía colombiana determinada mediante la isoterma de Langmuir. Suelos Ecuatoriales, v.9, n.1, p.15-20, 1978.

Berkowitz, J.; Anderson, M. A.; Amrhein, C. Influence of aging on phosphorus sorption to alum floc in lake water. Water Research, v.40, n.5, p.911-916, 2006.

Berkowitz, J.; Anderson, M. A.; Graham, R.C. Laboratory investigation of aluminum solubility and solid-phase properties following alum treatment of lake waters. Water Research, v.39, n.16, p.3918-3928, 2005.

Bohn, H.; Mc Neal, B. O’Connor, G. Química del suelo. 1.ed. México: Editorial Limusa - Noriega, 1993. 370p.

Borggaard, O. K.; Raben-Lange, B.; Gimsing, A. L.; Strobel, B. W. Influence of humic substances on phosphate adsorption by aluminium and iron oxides. Geoderma, v.127, n.3-4, p.270-279, 2005.

Bornemisza, E. Introducción a la química de suelos. Washington: OEA. 1982, 74p.

Cepeda, J. M.; Narro, E. A. Uso de isotermas de adsorción de fósforo para estimar los requerimientos de fertilizantes fosfatados en el cultivo de papa (Solanun tuberosum L.) en un suelo calcáreo. Agraria, v.2, n.1, p.138-154, 1986.

Das, J.; Patra, B. S.; Baliarsingh, N.; Parida, K.M. Adsorption of phosphate by layered double hydroxides in aqueous solutions. Applied Clay Science, v.32, n.3-4, p.252-260, 2006.

Fassbender, H. La adsorción de fosfatos en suelos fuertemente ácidos y su evaluación usando la isoterma de Langmuir. Fitotecnia Latinoamericana, v.3, n.1-2, p.203-216, 1966.

Fassbender, H.; Bornemisza, E. Química de suelos con énfasis en suelos de América Latina. 2.ed. Costa Rica: IICA. 1987, 420p.
Fox, R. L.; Kamprath, E. J. Phosphate sorption isotherms for evaluating the phosphate requirements of soils. Soil Science Society of America Proceedings, v.34, p.902-907, 1970.

Guggenberger, G.; Haumaier, L.; Thomas, R.; Zech, W. Assessing the organic phosphorus status of an Oxisol under tropical pastures following Savanna, using 31P NMR spectroscopy. Biology and Fertility of Soils, v.23, n.3, p.332-339, 1996.

Havlin, J. L.; Beaton, J. D.; Tisdale, S. L.; Nelson, W. L. Soil fertility and fertilizers. An introduction to nutrient management. 6.ed., New Jersey: Prentice Hall, 1999. 449p.

Hue, N. V.; Amien, I. Aluminum detoxification with green manures. Communications in Soil Science and Plant Analysis, v.20, n.15/16, p.1499-1511, 1989.

Hunter, D. J.; Yapa, L. G. G.; Hue, N. V. Effects of green manure and coral lime on corn growth and chemical properties of an acid Oxisol in Western Samoa. Biology and Fertility of Soils, v.24, n.3, p.266-273, 1997.

Liu, Y. Some consideration on the Langmuir isotherm equation. Colloids and Surfaces A: Physicochemical and Engineering Aspects, v.274, n.1-3, p.34-36, 2006.

Mejía, L. Génesis y características de los Oxisoles y suelos óxicos de los llanos orientales de Colombia y su relación con la fertilidad. Suelos Ecuatoriales, v.26, n.1, p.7-32, 1996.

Mota, M.; Rodríguez, T.; Montenegro, H.; Marulanda, S.; Correa, C.; Bendeck, M. Métodos analíticos del laboratorio de suelos. 5.ed. Bogotá: IGAC. 1990, 520p.

Murphy, J.; Riley, J. P. A modified single solution method for the determination of phosphate in natural waters. Analytica Chimica Acta, v.27, p.31-36, 1962.

Olsen, S. R.; Khasawneh, F. E. Use and limitations of physical-chemical criteria for assessing the status of phosphorus in soils. In: Khasawneh, F.E.; Sample E.C.; Kamprath E.J. (ed.) Madison: American Society of Agronomy, 1980. p.361-410.

Polyzopoulos, N. A.; Keramidas V. A.; Kiosse, H. Phosphate sorption by some alfisoles of Greece as described by commonly used isotherms. Soil Science Society of America Journal, v.49, p.81-84, 1985.

Porta, J.; López-Acebedo, M.; Roquero de Laburu, C. Edafología para la agricultura y el medio ambiente. Madrid: MundiPrensa, 1994. 929p.

Zhou, A.; Tang, H.; Wang, D. Phosphorus adsorption on natural sediments: Modeling and effects of $\mathrm{pH}$ and sediment composition. Water Research, v.39, n.7, p.1245-1254, 2005. 